\title{
Comparison of Rheological, Drug Release, and Mucoadhesive Characteristics upon Storage between Hydrogels with Unmodified or Beta-Glycerophosphate-Crosslinked Chitosan
}

\author{
Emilia Szymańska (D), Anna Czajkowska-Kośnik (D), and Katarzyna Winnicka (D) \\ Department of Pharmaceutical Technology, Faculty of Pharmacy, Medical University of Biatystok, Mickiewicza 2c, \\ Bialystok 15-222, Poland \\ Correspondence should be addressed to Emilia Szymańska; esz@umb.edu.pl
}

Received 19 April 2018; Revised 25 July 2018; Accepted 15 August 2018; Published 19 September 2018

Academic Editor: Mingqiang Li

Copyright ( $) 2018$ Emilia Szymańska et al. This is an open access article distributed under the Creative Commons Attribution License, which permits unrestricted use, distribution, and reproduction in any medium, provided the original work is properly cited.

\begin{abstract}
The physicochemical characteristics of beta-glycerophosphate-crosslinked chitosan hydrogels were investigated upon long-term storage at ambient, accelerated, and refrigerated conditions and compared to unmodified chitosan formulations. Additionally, the impact of chitosan modification on the ex vivo mucoadhesive performance in contact with porcine vaginal mucosa and on the drug release profile from hydrogels was evaluated. Viscosity and mechanical properties of formulations with unmodified chitosan decreased significantly upon storage regardless of tested conditions as a result of hydrolytic depolymerization. Introduction of ion crosslinker exerted stabilizing effect on physicochemical performance of chitosan hydrogels but only upon storage at refrigerated conditions. Beta-glycerophosphate-modified chitosan formulations preserved organoleptic, rheological behavior, and hydrogel structure up to 3 -month storage at $4 \pm 2^{\circ} \mathrm{C}$. Viscosity variations upon storage influenced markedly mucoadhesive properties and drug release rate from hydrogels.
\end{abstract}

\section{Introduction}

Application of multifunctional polymers as effective excipients in drug delivery systems is a current and attractive concept in pharmaceutical technology [1]. Among various polymer materials, chitosan (CS) - an abundantly accessible polysaccharide, owing to its biodegradability and biocompatibility is extensively investigated for a number of pharmaceutical and medical applications, comprising prolonged drug release delivery systems [2], wound dressings [3], cartilage and bone tissue engineering scaffolds $[4,5]$, nanocarriers for gene delivery [6], or vaccine adjuvants [7].

CS is commercially possessed by deacetylation of chitin derived mainly from the exoskeleton of crustaceans, though alternative production methods by fungal fermentation have been also explored to provide CS with more defined characteristics [8]. Due to polycationic behavior, CS structure can be certainly modified and utilized into diverse semisolid or solid vehicles. Hence, CS may be regarded to be a promising tool not only in pharmaceutical [9] but also in cosmetic [10] or food industry [11].

CS displays mucoadhesive potential enabling interaction with mucin via electrostatic or hydrogen bonding. Mucoadhesiveness, providing an intimate contact between CS-based dosage form and mucosal tissue, appears to be beneficial, especially in terms of CS application in technology of drug carriers for local: nasal [12], ocular [2], buccal [13], or vaginal delivery [14]. Additionally, with regard to capability of swelling and creating hydrogel matrix, CS may be useful in the development of modified release delivery systems $[15,16]$. In recent years, particular attention has been directed to the use of CS as an antimicrobial adjunctive $[17,18]$. This capability of increasing the pharmacological action of an antimicrobial agent provides the opportunity for combination therapy in which CS acts as the effective excipient and simultaneously as an active part of the treatment. 
Regarding abovementioned advantages of CS, experimental research was conducted to investigate CS usability as pharmaceutical excipient in technology of vaginal hydrogels for the treatment of mycotic vaginosis. Designed semisolid platforms displayed favorable ability to interact with porcine vaginal mucosa confirmed in ex vivo studies followed with enhanced anti-Candida activity compared to commercially available preparation with clotrimazole $[19,20]$. Nonetheless, because of fast and uncontrollable disintegration in an acidic environment and poor rheological behavior with regard to vaginal administration, further improvement of CS structure was needed. Previously presented data showed that CS crosslinked with beta-glycerophosphate disodium (bGP) demonstrated a promising potential for the development of mucoadhesive hydrogels with feasible rheological and mechanical properties [20].

Even though CS is regarded as a multifunctional compound in the technology of drug carriers, only a limited number of pharmaceutical products with CS (apart from hemostatic dressings, products for wound healing, or nutraceuticals) are commercially available. One of the main drawbacks, limiting the application of CS in pharmaceutical technology, is its susceptibility to environmental conditions (especially with regard to humidity and temperature) as well as processing factors, which in consequence might impact the physicochemical and pharmaceutical performance of polymer's dosage form upon storage [21]. According to the literature, modification of CS structure via grafting, chemical, or ionic crosslinking may overcome the problems of reduced long-term stability of polymer-based drug delivery systems [22-24]. Since, to our best knowledge, only limited number of studies have been devoted to the issue of long-term stability data of CS-based dosage forms, therefore, the goal of the current paper was to examine the influence of crosslinking with bGP on the physicochemical performance of CS hydrogels with a model antifungal agent upon storage. Designed semisolid platforms with bGP/CS were characterized by organoleptic, rheological, mucoadhesive properties and compared to unmodified CS-based hydrogels. The impact of CS modification on the drug release profile from hydrogels upon storage was also examined.

\section{Materials and Methods}

2.1. Materials. High-quality medium molecular weight CS (200-400 kDa; viscosity $370-390 \mathrm{mPa} \cdot \mathrm{s}$ at $25^{\circ} \mathrm{C}, 1 \%(w / w)$ in $1 \%(v / v)$ acetic acid) with deacetylation degree $80 \%$ was obtained from Heppe Medical CS GmbH (Haale, Germany). Clotrimazole was a gift from Ziaja Ltd. (Gdańsk, Poland). bGP disodium salt hydrate and Cremophor EL were obtained from Sigma-Aldrich (Steinheim, Germany). Acetic acid $80 \%$, glycerolum $85 \%$, disodium hydrogen phosphate, potassium dihydrogen phosphate, sodium acetate, and sodium hydroxide were purchased from Chempur (Piekary Śląskie, Poland). Methanol (HPLC grade) was purchased from Merck (Darmstadt, Germany). Vaginal mucoadhesive gel Replens ${ }^{\mathrm{TM}}$ was acquired from APC Instytut Sp. z o.o. (Warsaw, Poland). Water for HPLC was distilled and passed through a reverse osmosis system Milli Q Reagent Water System (Billerica, MA, USA).

\subsection{Experimental Procedure}

2.2.1. Hydrogels with Unmodified or bGP-Crosslinked CS. Hydrogels with clotrimazole were prepared using mechanical stirrer according to the previously described technique [20]. Briefly, CS base with concentration of $3 \%$ or $4 \%(w / w)$ was obtained by dissolving proper amount of polymer in a gently heated acetic acid solution (the weight ratio CS : glacial acetic acid was $0.6: 1.0$ ) [25] and stirred until homogenous mixtures appeared. To prepare bGP-modified CS hydrogels, the appropriate amount (necessary to simulate vaginal $\mathrm{pH} 4.5$ ) of cold $45 \%(w / w)$ aqueous solution of bGP (the weight ratio CS to bGP 1:0.63) was added dropwise to the cold CS base with continuous agitation. Subsequently, suitable amount of unmodified CS or bGP/CS base was carefully added to a mixture of humectant, preservative, and clotrimazole solubilized in Cremophor EL to obtain uniform dispersion of drug in the hydrogel matrix. Hydrogels' composition is displayed in Table 1 .

2.2.2. Long-Term Evaluation of Hydrogels. Hydrogel formulations were subjected to long-term stability studies under defined conditions of humidity and temperature according to ICH guideline [26]. Briefly, hydrogels closed in sealed polyethylene containers and additionally in cardboard packages to prevent from light exposure were kept in humidity chambers (CTC 256 Memmert GmbH, Schwabach, Germany) maintained at $25 \pm 2{ }^{\circ} \mathrm{C} / 60 \pm 5 \%$ relative humidity $(\mathrm{RH})$ and $40 \pm 2^{\circ} \mathrm{C} / 70 \pm 5 \% \mathrm{RH}$ as well as in the refrigerator at $4 \pm 2^{\circ} \mathrm{C}$. At specific time intervals (displayed in Table 2), macroscopic performance, $\mathrm{pH}$ and viscosity values, drug particle's diameter, and drug content uniformity followed with mechanical and mucoadhesive performance were evaluated. Formulations were maintained for at least $1 \mathrm{~h}$ at ambient temperature prior analysis.

(1) Macroscopic and Microscopic Analysis. Organoleptic analysis in terms of consistency, odor, and color was performed in accordance to the European Pharmacopoeia (Eur. Ph.) by placing hydrogel in a thin layer on a glass slide [27].

Particles size analysis was accomplished using an optical microscope Motic BA 400 (Moticon, Wetzlar, Germany) with magnifications $100 \mathrm{x}$ and $400 \mathrm{x}$. Concisely, $0.5 \mathrm{mg}$ of each formulation (corresponding to $10 \mu \mathrm{g}$ of clotrimazole) was located on a glass slide and the longest dimension from edge to edge of drug particles was defined for particles present in at least three areas of observation [28].

(2) Viscosity Measurement. The apparent viscosity was measured according to Eur. Ph. [27] using a digital rotational Brookfield RVDV-III ULTRA Viscometer (Brookfield Engineering Laboratories, Germany) at ambient temperature. Each hydrogel sample $(0.5 \mathrm{~g})$ was placed in the thermostated sampler holder of the viscometer, allowed to equilibrate for $10 \mathrm{~min}$ at $25 \pm 1^{\circ} \mathrm{C}$, and then $\mathrm{C}-52$ spindle was lowered into 
TABLE 1: Composition of hydrogels with unmodified CS (F1, F2) or bGP-crosslinked CS (F3, F4).

\begin{tabular}{|c|c|c|c|c|c|}
\hline \multirow{3}{*}{ Ingredient (g) } & \multirow{3}{*}{ Category } & \multicolumn{4}{|c|}{ Hydrogel with } \\
\hline & & \multicolumn{2}{|c|}{ Unmodified CS } & \multicolumn{2}{|c|}{ bGP-modified CS } \\
\hline & & F1 & F2 & F3 & $\mathrm{F} 4$ \\
\hline CS & Gelling agent & 3.0 & 4.0 & 3.0 & 4.0 \\
\hline bGP $45 \%(w / w)$ & Ion crosslinker & - & - & 4.2 & 5.6 \\
\hline Clotrimazole & API & 2.0 & 2.0 & 2.0 & 2.0 \\
\hline Glycerolum & Humectant & 5.0 & 5.0 & 5.0 & 5.0 \\
\hline Cremophor EL & Solubilizer & 6.0 & 6.0 & 6.0 & 6.0 \\
\hline D-Glucono-1,5-lactone and sodium benzoate & Preservative & 1.0 & 1.0 & 1.0 & 1.0 \\
\hline Acetic acid & Vehicle & Up to 100.0 & Up to 100.0 & Up to 100.0 & Up to 100.0 \\
\hline
\end{tabular}

TABLE 2: Time intervals applied for stability studies [26].

\begin{tabular}{lccc}
\hline $\begin{array}{l}\text { Stability } \\
\text { studies }\end{array}$ & $\begin{array}{c}\text { Storage } \\
\text { conditions }\end{array}$ & $\begin{array}{c}\text { The minimum } \\
\text { period of storage }\end{array}$ & $\begin{array}{c}\text { Time intervals } \\
\text { (months) }\end{array}$ \\
\hline Long term & $\begin{array}{c}25 \pm 2^{\circ} \mathrm{C} / 60 \\
\pm 5 \% \mathrm{RH} \\
4 \pm 2^{\circ} \mathrm{C}\end{array}$ & $\begin{array}{c}12 \text { months } \\
6 \text { months }\end{array}$ & $1,3,6,12$ \\
\hline Accelerated & $\begin{array}{c}40 \pm 2^{\circ} \mathrm{C} / 75 \\
\pm 5 \% \mathrm{RH}\end{array}$ & 6 months & $1,3,6$ \\
\hline
\end{tabular}

the sample. The shear rate was 6/s. Each experiment was performed at least three times.

(3) $\mathrm{pH}$ Determination. The $\mathrm{pH}$ of hydrogels was estimated by a glass electrode of the $\mathrm{pH}$ meter Orion-3 Star (Thermo Scientific, Waltham, MA, USA) in triplicate.

(4) Drug Content Uniformity. Drug content uniformity was evaluated after the extraction of hydrogel samples with absolute ethanol, centrifugation at $4000 \mathrm{rpm}$ for $20 \mathrm{~min}$, and filtration through $0.45 \mu \mathrm{m}$ nylon filters. Samples were next suitably diluted with mobile phase and determined for clotrimazole content by HPLC method according to [19]. Separations were performed at ambient temperature on $5 \mu \mathrm{m}$ Zorbax Eclipse XDB-C18 $(4.6 \mathrm{~mm} \times 150 \mathrm{~mm})$ column (Agilent, Waldbronn, Germany). The mobile phase consisted of methanolphosphate buffer pH7.4 $(4: 1, v / v)$, the flow rate was $1.0 \mathrm{~mL} / \mathrm{min}$, and the retention time was $5.3 \mathrm{~min}$. The detector was set to $210 \mathrm{~nm}$. The standard calibration curve was linear over the range of 1 to $100 \mu \mathrm{g} / \mathrm{mL}\left(R^{2}=0.995\right)$.

(5) Mechanical Properties. Mechanical properties were evaluated using Texture Analyser TA.XT Plus (Stable Micro System, UK) equipped with a backward extrusion measuring system $\mathrm{A} / \mathrm{BE}$ ( $25 \mathrm{~mm}$ diameter). Each preparation $(30.0 \pm 0.2 \mathrm{~g})$ was located in a measuring vessel, and then a disc was compressed with a speed of $2 \mathrm{~mm} / \mathrm{s}$ into the sample to a defined depth of $5 \mathrm{~mm}$. The experimental parameters were established during preliminary study. All measurements were carried out at $25 \pm 2^{\circ} \mathrm{C}$. From the force-time curves, the values of consistency were calculated and the hardness parameter was recorded using Texture
Exponent 32 software. Each experiment was carried out at least three times.

(6) Ex Vivo Mucoadhesive Properties. Ex vivo mucoadhesive behavior of hydrogels in contact with porcine vaginal mucosa was evaluated according to a method defined previously [15] with using Texture Analyser TA.XT. Plus (Stable Micro Systems, Godalming, UK) equipped with the measuring system A/GMP. Briefly, freshly excised porcine vaginal mucosa was adhered by double-sided tape to the platform below the texture analyzer probe whilst hydrogel sample $(1.5 \mathrm{~mL})$ was set on the upper probe and secured with the attached support collar. The experiments were conducted at $37 \pm 1^{\circ} \mathrm{C}$. Prior to analysis, the support collar was removed and the probe was lowered onto the surface of the porcine vaginal mucosa with a speed of $0.5 \mathrm{~mm} / \mathrm{s}$. After keeping a contact time for $180 \mathrm{~s}$ with contact force $0.5 \mathrm{~N}$, the surfaces were separated at a constant rate of $0.1 \mathrm{~mm} / \mathrm{s}$. The maximum detachment force $\left(F_{\max }\right)$ as a function of displacement was recorded directly from Texture Exponent 32 software, and the work of adhesion $\left(W_{a d}\right)$ was calculated from the area under the force vs. distance curve. Each experiment was performed six times.

(7) In Vitro Drug Release Studies. Due to unfavorable organoleptic and rheological changes observed for hydrogels upon their storage at ambient and accelerated conditions, in vitro dissolution studies were performed only for formulations with preserved rheological and mechanical behavior upon 6-month storage at $4 \pm 2^{\circ} \mathrm{C}$. Drug release was measured through natural cellulose membrane (Cuprophan with MWCO $10000 \mathrm{Da}$, Medicell, London, UK) using an Enhancer cell (Agilent Technologies, Cary, USA) [29] and USP dissolution Apparatus II (Agilent 708-DS, Agilent Technologies, Cary, USA). Each formulation ( $1.0 \mathrm{~g})$ was placed in the drug reservoir on the top of the membrane making certain that no entrapped air was present at the interface of the dosage form and the membrane (diffusion area $3.80 \mathrm{~cm}^{2}$ ). The dissolution medium was $100 \mathrm{~mL}$ acetic buffer ( $\mathrm{pH} 4.5$ ) with addition of $1 \%$ surfactant to retain sink conditions. The temperature of medium was maintained at 37 $\pm 1^{\circ} \mathrm{C}$, and a stirring rate was $75 \mathrm{rpm}$. Samples $(2 \mathrm{~mL})$ were withdrawn at the predetermined time points, filtered through $0.45 \mu \mathrm{m}$ CA paper filters, diluted with mobile phase, and 
quantified using HPLC method. Withdrawn samples were replaced with equal volume of fresh medium. All release experiments were carried out in triplicate.

2.3. Statistical Analysis. Quantitative variables were expressed as the mean \pm standard deviation (SD) or the median by using MS Excel software. A statistical analysis of mucoadhesive measurements was accomplished using a nonparametric Kruskal-Wallis test, with the Statistica 12.0 software. Measurements were considered significant at $p<0.05$.

\section{Results and Discussion}

3.1. Characterization of Hydrogels upon Storage. Stability evaluation provides the critical characteristics of the drug dosage form in terms of its physicochemical or biopharmaceutical properties, and thus it is crucial for the full assessment of product quality during preformulation analysis. Basically, for drug products, a comparative stability data in both long term (at $25^{\circ} \mathrm{C} / 60 \% \mathrm{RH}$ for 12 months) and accelerated conditions $\left(40^{\circ} \mathrm{C} / 75 \% \mathrm{RH}\right)$ is required to define an acceptable product shelf-life [26]. Given these premises, the effect of ion crosslinking on CS hydrogel physicochemical characteristics upon storage at $25^{\circ} \mathrm{C} / 60 \% \mathrm{RH}$ and $40^{\circ} \mathrm{C} /$ $75 \% \mathrm{RH}$ was studied. Regarding the fact that CS is recommended to store at low temperatures $\left(2-8^{\circ} \mathrm{C}\right)$ [27], additional examination of formulations upon storage at $4 \pm 2^{\circ} \mathrm{C}$ was also provided.

Directly after preparation, all hydrogels possessed smooth, uniform consistency and off-white color resulting from the presence of suspended clotrimazole (Figure S1). Significant organoleptic differences were noticed upon first months of storage at ambient conditions. Hydrogels with unmodified CS (F1, F2) became yellowish within one month at $25^{\circ} \mathrm{C} / 60 \% \mathrm{RH}$, and the color alterations were deepened over time. After 3-month storage, loss of gel structure and homogeneity of formulations F1 and F2 was observed. It should be noted that hydrogels with bGP-modified CS (F3 and F4) remained stable in terms of organoleptic properties within 3-month storage. Nonetheless, upon 6-month storage at $25^{\circ} \mathrm{C} / 60 \% \mathrm{RH}$, a substantial liquidation with visible drug particle sedimentation and simultaneous color changes occurred in all examined hydrogels (Figure S2).

The most profound and particularly fast alterations in hydrogel performance appeared upon their storage at $40^{\circ} \mathrm{C} /$ $75 \% \mathrm{RH}$. When hydrogels with unmodified chitosan (F1 and F2) were subjected to accelerated stability studies, loss of homogeneity follow with color changes and drug particle sedimentation occurred just upon 1-month storage period. Regarding formulations with bGP/CS (F3 and F4), a significant enhancement in the structure stiffness was observed over time and after 3 months at $40^{\circ} \mathrm{C} / 75 \% \mathrm{RH}$, hydrogels transformed into brown solid state formulations (Figure S3). The observed time-delayed sol/gel transition was probably attributed to thermosensitivity of CS in the presence of ion crosslinker, which affected the time and the temperature of CS gelling [30].

Notably, all hydrogel formulations remained organoleptically stable up to 3 months at refrigerated conditions. Upon their 6-month storage at $4 \pm 2^{\circ} \mathrm{C}$, no alterations in bGP/CS hydrogels' appearance were observed whereas substantial changes in consistency with simultaneous drug particle sedimentation in hydrogels with unmodified CS (F1 and F2) occurred (Figure S4).

The microscopic analysis of all investigated formulations revealed no considerable change in average particle size of suspended clotrimazole upon storage at refrigerated conditions although the presence of few aggregates of drug particles was observed in hydrogels F1 and F2 (Figure 1).

Due to the presence of undesirable changes in hydrogels' organoleptic properties followed with loss of their homogeneity upon 3-month and 6-month storage at accelerated or ambient conditions (Figures S2 and S3), respectively, formulations F1-F4 were excluded from further long-term analysis. Evaluation of drug content uniformity was performed for these hydrogel formulations, which preserved their viscosity and organoleptic properties upon storage. As it is presented in Table 3, clotrimazole was found to be chemically stable in hydrogels F1 and F2 upon 3-month storage at ambient and refrigerated conditions and upon 1-month at $40^{\circ} \mathrm{C} / 75 \% \mathrm{RH}$. Regarding bGP/CS hydrogels, the drug content was found to be uniform and was within acceptable USP limit 90\%-110\% after storage in all investigated storage conditions [31].

A slight increase in $\mathrm{pH}$ values of hydrogels $\mathrm{F} 1$ and $\mathrm{F} 2$ was observed after 3-month storage at $40^{\circ} \mathrm{C} / 75 \% \mathrm{RH}$ and 6month at $25^{\circ} \mathrm{C} / 60 \% \mathrm{RH}$ in contrast to formulations $\mathrm{F} 3$ and $\mathrm{F} 4$, which displayed decreasing $\mathrm{pH}$ trend upon storage regardless of temperature conditions (Table 3). Nonetheless, at the end of the stability studies, all hydrogels displayed the $\mathrm{pH}$ values below 4.5 , which was maintained within the physiological range 3.5-4.9 [32].

3.2. Viscosity and Mechanical Measurements. To assess the influence of ion crosslinking on the rheological behavior of CS hydrogels upon storage, the viscosity measurements were performed. The intrinsic viscosity of CS defines the ability to form viscous solution and is proportional to the polymer average molecular weight [33]. Figure 2 displays the viscosity variations upon storage at different conditions.

Freshly prepared hydrogels F3 and F4 displayed lower viscosities compared to unmodified CS formulations (F1 and F2) most likely as a consequence of greater ionic strength resulted from the introduction of bGP. All formulations exhibited non-Newtonian pseudoplastic behavior, and the presence of bGP improved thixotropic properties of CS hydrogels [20].

It can be seen that viscosity of hydrogels with unmodified CS (F1 and F2) decreased significantly over time regardless of tested conditions. Formulation F2, with higher concentration of CS, displayed more profound viscosity variations and loss of more than $40 \%, 50 \%$, and $90 \%$ of the initial value after 3month storage at $4 \pm 2^{\circ} \mathrm{C}, 25^{\circ} \mathrm{C} / 60 \% \mathrm{RH}$, and $40^{\circ} \mathrm{C} / 75 \% \mathrm{RH}$ was observed, respectively. Similarly, Chattopadhyay and Inamdar observed the impact of CS concentration on the viscosity of polymer solution over storage [34]. Interestingly, the presence of ion crosslinker exerted stabilizing effect on CS hydrogels' viscosity but only upon their storage at 


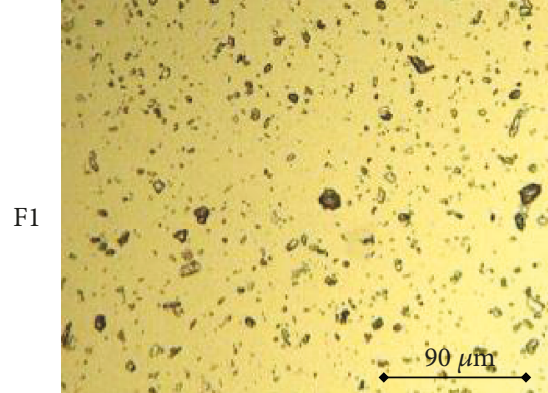

(a)

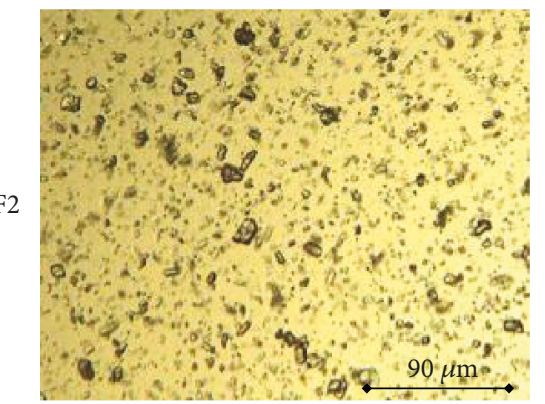

(b)

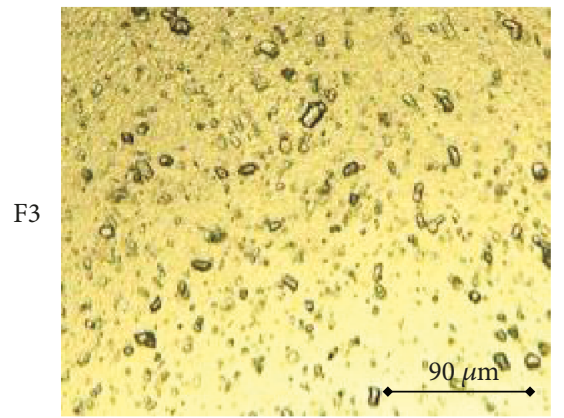

(c)

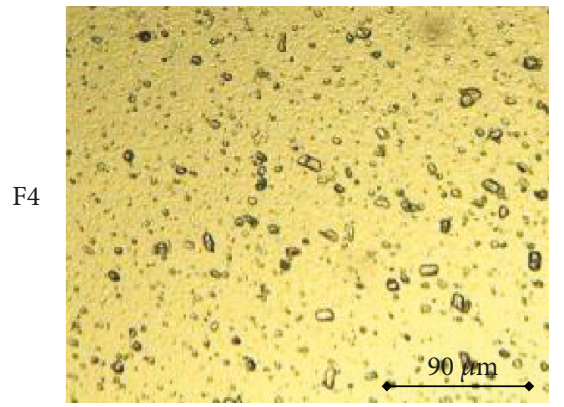

(d)

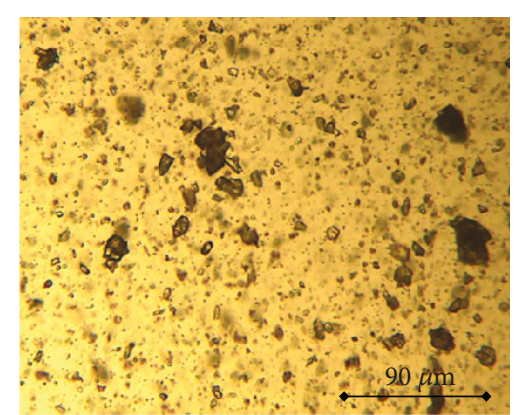

(e)

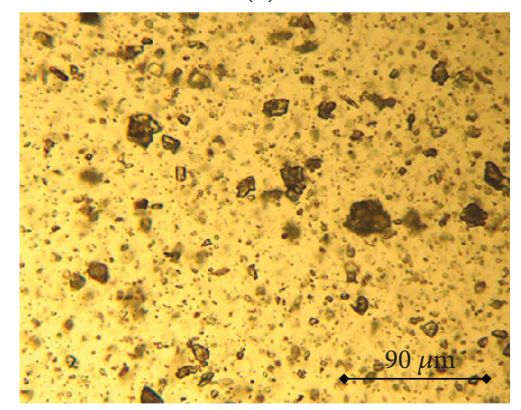

(f)

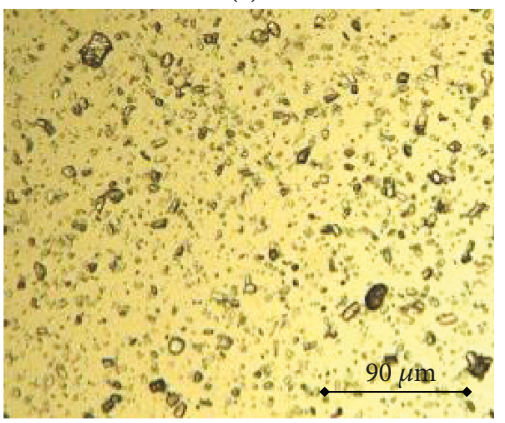

(g)

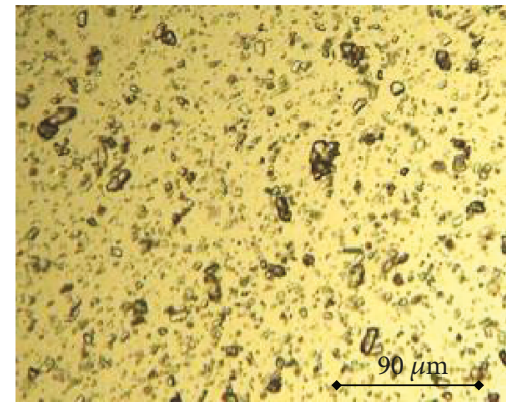

(h)

Figure 1: The optical images of hydrogels with unmodified CS (F1, F2) or with bGP-crosslinked CS (F3, F4) after preparation (a-d) and upon 3-month (F1, F2) (e-f) or 6-month storage (g-h) at refrigerated conditions (F3, F4).

refrigerated conditions. After 3 months at $4 \pm 2^{\circ} \mathrm{C}$, hydrogels F3 and F4 displayed comparable viscosity values to those obtained for freshly prepared formulations (Figure 2(c)). Nonetheless, after a period of 6 months, a drop of more than $15 \%$ and $25 \%$ in viscosity parameter of formulations F3 and F4 was noticed, respectively. In contrast, profound viscosity variations of bGP/CS hydrogels were noticed upon their storage at ambient and accelerated conditions. An increase in viscosity values and solidification of formulations F3 and F4 was obtained within a period of 3 months at $40^{\circ} \mathrm{C} / 75 \% \mathrm{RH}$ (Figure 2(b)). Conversely, a slight decrease in viscosity parameter upon their 3-month storage at $25^{\circ} \mathrm{C} / 60 \% \mathrm{RH}$ was noticed and after 6 months, a significant drop in viscosity of bGP/CS hydrogels (approximately $40 \%$ of the initial value) was recorded.

The substantial viscosity alterations observed particularly in hydrogels with unmodified CS may be attributed to partial degradation of CS in organic acid solution $[33,35,36]$. In 
TABLE 3: Changes in organoleptic properties, clotrimazole content, and drug particle size in hydrogels with unmodified CS (F1, F2) or with bGP-crosslinked CS (F3, F4) upon storage compared to values obtained to freshly prepared formulations.

\begin{tabular}{|c|c|c|c|c|c|}
\hline \multirow{3}{*}{ Storage time (months) } & \multirow{3}{*}{ Parameter } & \multicolumn{4}{|c|}{ Hydrogel with } \\
\hline & & \multicolumn{3}{|c|}{ Unmodified CS } & \multirow{2}{*}{$\begin{array}{l}\text { bGP-crosslinked CS } \\
\text { F4 }\end{array}$} \\
\hline & & $\mathrm{F} 1$ & $\mathrm{~F} 2$ & F3 & \\
\hline \multirow{3}{*}{0} & $\mathrm{pH}$ & $4.06 \pm 0.02$ & $4.08 \pm 0.01$ & $4.54 \pm 0.04$ & $4.56 \pm 0.03$ \\
\hline & Particle size range $(\mu \mathrm{m})$ & $6.0-49.5$ & $6.0-47.0$ & $4.0-40.0$ & $4.0-41.5$ \\
\hline & Drug content $(\%)^{*}$ & $95.6 \pm 4.9$ & $98.2 \pm 3.5$ & $97.3 \pm 4.1$ & $97.4 \pm 3.3$ \\
\hline \multicolumn{6}{|l|}{$25^{\circ} \mathrm{C} / 60 \% R H$} \\
\hline \multirow{3}{*}{1} & $\mathrm{pH}^{*}$ & $4.16 \pm 0.05$ & $4.23 \pm 0.05$ & $4.51 \pm 0.04$ & $4.53 \pm 0.02$ \\
\hline & Particle size range $(\mu \mathrm{m})$ & $7.5-52.5$ & $7.5-57.0$ & $6.0-29.5$ & $5.0-48.5$ \\
\hline & Drug content $(\%)^{*}$ & $94.8 \pm 3.3$ & $96.1 \pm 4.2$ & $96.5 \pm 3.5$ & $97.1 \pm 3.9$ \\
\hline \multirow{3}{*}{3} & $\mathrm{pH}^{*}$ & $4.40 \pm 0.02$ & $4.38 \pm 0.03$ & $4.35 \pm 0.02$ & $4.27 \pm 0.03$ \\
\hline & Particle size range $(\mu \mathrm{m})$ & $7.0-58.0$ & $6.0-54.5$ & $4.0-41.0$ & $4.0-34.5$ \\
\hline & Drug content $(\%)^{*}$ & $94.6 \pm 5.9$ & $92.4 \pm 5.1$ & $98.1 \pm 4.1$ & $95.2 \pm 4.5$ \\
\hline \multirow{3}{*}{6} & $\mathrm{pH}^{*}$ & $4.31 \pm 0.02$ & $4.39 \pm 0.01$ & $4.26 \pm 0.02$ & $4.23 \pm 0.02$ \\
\hline & Particle size range $(\mu \mathrm{m})$ & n.d. & n.d. & Sedimentation & Sedimentation \\
\hline & Drug content $(\%)^{*}$ & n.d. & n.d. & n.d. & n.d. \\
\hline \multicolumn{6}{|l|}{$40^{\circ} \mathrm{C} / 75 \% \mathrm{RH}$} \\
\hline \multirow{3}{*}{1} & $\mathrm{pH}^{*}$ & $4.20 \pm 0.03$ & $4.24 \pm 0.05$ & $4.56 \pm 0.04$ & $4.51 \pm 0.02$ \\
\hline & Particle size diameter $(\mu \mathrm{m})$ & Sedimentation & Sedimentation & $10.0-53.0$ & $5.0-61.0$ \\
\hline & Drug content $(\%)^{*}$ & n.d. & n.d. & $96.5 \pm 3.5$ & $97.1 \pm 3.9$ \\
\hline \multirow{3}{*}{3} & $\mathrm{pH}^{*}$ & $4.40 \pm 0.02$ & $4.48 \pm 0.02$ & $4.36 \pm 0.01$ & $4.29 \pm 0.03$ \\
\hline & Particle size range $(\mu \mathrm{m})$ & n.d. & n.d. & $5.0-44.5$ & $4.5-37.0$ \\
\hline & Drug content $(\%)^{*}$ & n.d. & n.d. & $93.1 \pm 7.1$ & $94.2 \pm 6.8$ \\
\hline \multicolumn{6}{|l|}{$4 \pm 2^{\circ} \mathrm{C}$} \\
\hline \multirow{3}{*}{1} & $\mathrm{pH}^{*}$ & $4.10 \pm 0.05$ & $4.03 \pm 0.05$ & $4.56 \pm 0.04$ & $4.53 \pm 0.02$ \\
\hline & Particle size range $(\mu \mathrm{m})$ & $12.0-58.5$ & $7.5-65.0$ & $8.0-59.0$ & $10.5-45.0$ \\
\hline & Drug content $(\%)^{*}$ & $94.3 \pm 3.8$ & $96.1 \pm 4.2$ & $96.5 \pm 3.5$ & $97.1 \pm 3.9$ \\
\hline \multirow{3}{*}{3} & $\mathrm{pH}^{*}$ & $4.10 \pm 0.02$ & $4.12 \pm 0.02$ & $4.31 \pm 0.02$ & $4.37 \pm 0.03$ \\
\hline & Particle size range $(\mu \mathrm{m})$ & Sedimentation & $6.0-59.5$ & $11.0-40.0$ & $5.0-45.5$ \\
\hline & Drug content $(\%)^{*}$ & n.d. & $95.1 \pm 8.4$ & $98.1 \pm 4.7$ & $95.2 \pm 4.5$ \\
\hline \multirow{3}{*}{6} & $\mathrm{pH}^{*}$ & $4.23 \pm 0.03$ & $4.29 \pm 0.03$ & $4.28 \pm 0.03$ & $4.37 \pm 0.01$ \\
\hline & Particle size range $(\mu \mathrm{m})$ & n.d. & n.d. & $6.5-46.0$ & $5.0-40.0$ \\
\hline & Drug content $(\%)^{*}$ & n.d. & n.d. & $94.1 \pm 4.9$ & $96.0 \pm 3.7$ \\
\hline
\end{tabular}

${ }^{*} n=3$, mean \pm SD; n.d.: not determined.

overall, storage temperature and CS concentration were found to accelerate the rate of hydrolysis of unmodified CS $[33,34]$. Our findings from stability analysis at refrigerated conditions are in the agreement with studies accomplished by Nguyen et al., which revealed that the ratio of CS decomposition in acetic acid environment could be reduced upon storage at $4 \pm 2^{\circ} \mathrm{C}$ [35]. In contrast, RuelGariépy et al. observed poor stability of bGP/CS thermogelling solutions below room temperature as gelation of the systems occurred upon 3-month storage at refrigerated conditions [37]. Similarly, Supper et al. indicated that the CS/bGP thermogelling solutions intended for parenteral administration possessed reduced physicochemical stability both under ambient and refrigerated conditions [38]. Nonetheless, in the present studies, much higher CS concentration was used for hydrogel preparation (3-4\%) as compared to the abovementioned studies, and it could be regarded as one of the factor improving hydrogel stability. In addition, the ability of bGP to balance the effect of the presence of acetic acid solution might have slowed down the rate of CS degradation and in consequence maintained the viscosity parameters over storage.

To assess deeper insight into the internal structure of prepared hydrogels upon their storage, the mechanical properties: hardness and consistency-parameters reflecting the degree of difficulty in loss of initial formulations' structure, 


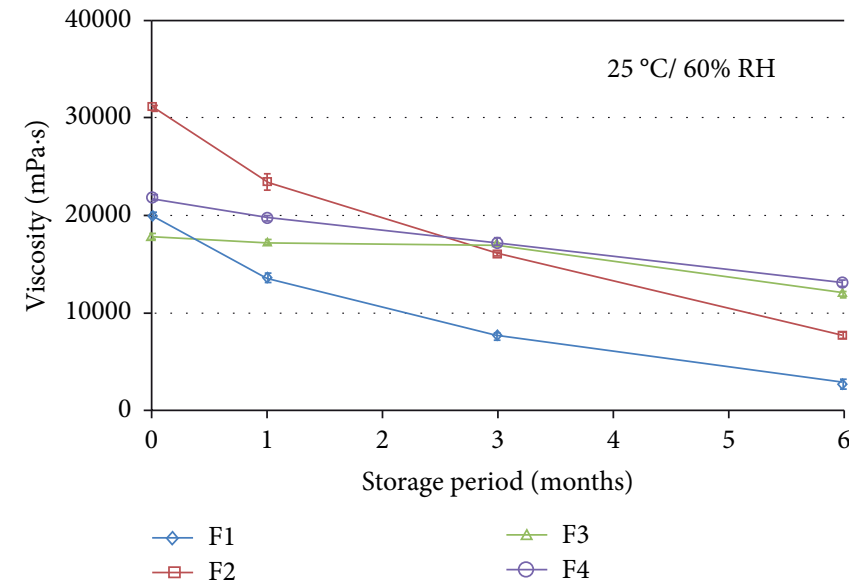

(a)

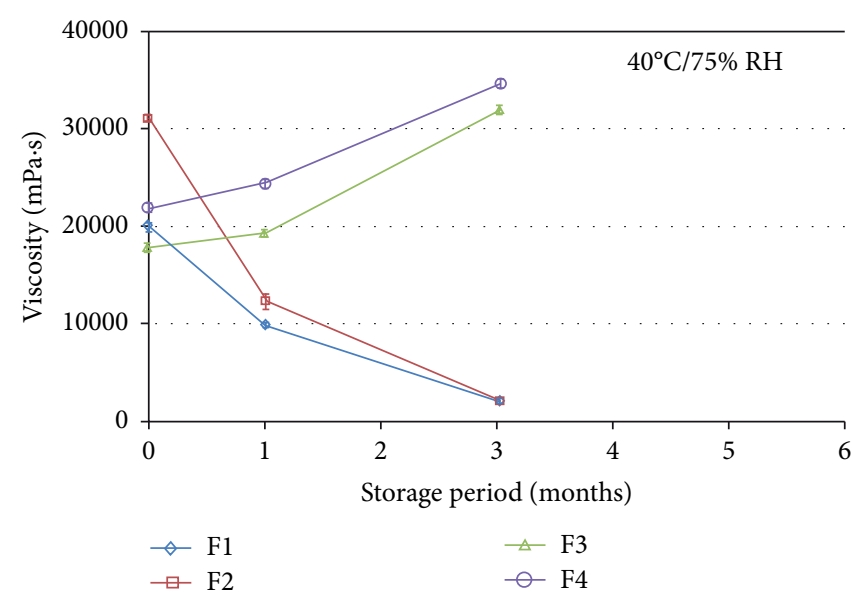

(b)

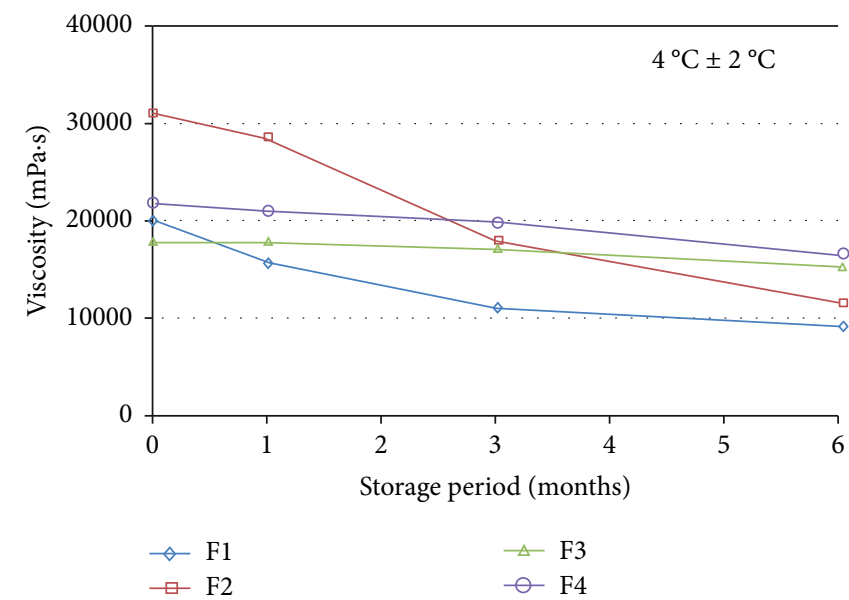

(c)

Figure 2: Variations in viscosity values of hydrogels with unmodified CS (F1, F2) or with bGP-crosslinked CS (F3, F4) upon storage at ambient (a), accelerated (b), and refrigerated conditions (c); $n=3$, mean \pm SD.

were examined (Figures 3(a)-3(f)). A correlation between investigated mechanical performance and hydrogel viscosity was displayed (Figure 2).

Noticeable drop in hardness and consistency values upon storage in all tested conditions was observed for hydrogels with unmodified CS (F1 and F2). Hydrogels with bGP/CS preserved their mechanical properties upon storage at 4 $\pm 2^{\circ} \mathrm{C}$ up to 6 months. However, differences in structural behavior were recorded when formulations F3 and F4 were subjected to stability studies under accelerated and ambient conditions. Loss of bGP/CS hydrogels' hardness (more than $30 \%$ of the initial values) was noticed upon 6 -month storage at $25^{\circ} \mathrm{C} / 60 \% \mathrm{RH}$ whereas substantially thicker and firmer structure of formulations $\mathrm{F} 3$ and $\mathrm{F} 4$ after 3 months at $40^{\circ} \mathrm{C} /$ $75 \% \mathrm{RH}$ was observed.

3.3. Mucoadhesion Studies. Mucoadhesive tests were conducted for all hydrogel formulations stored for up to six months and displayed in Figure 4. Because of profound loss of homogeneity followed with a major drop in viscosity values (which hinder the ability to maintain in a support collar of mucoadhesive probe), formulations F1 and F2 were excluded from mucoadhesive measurements at accelerated conditions as well as upon 3- and 6-month storage at ambient and refrigerated conditions, correspondingly.

Results obtained from ex vivo mucoadhesive measurements upon storage clearly displayed that the ability to adhere to porcine vaginal mucosa differed significantly between unmodified and ion-crosslinked CS formulations. bGP/CS hydrogels stored at $4 \pm 2^{\circ} \mathrm{C}$ exhibited comparable mucoadhesive behavior compared to freshly prepared formulations within 3-month storage. However, after 6 months, a decrease in ability to adhere to porcine vaginal mucosa of bGP/CS hydrogels was noticed. Similarly, upon storage at ambient conditions, formulations with bGP/CS (F3 and F4) tended to lose their mucoadhesive properties after a period of 3 months. In contrast, a significant drop in $F_{\max }$ and $W_{\text {ad }}$ values $(p<0.05)$ of formulations with unmodified $\mathrm{CS}$ (F1 and F2) was recorded just after 1-month storage at $4 \pm 2^{\circ} \mathrm{C}$ and $25^{\circ} \mathrm{C} / 60 \% \mathrm{RH}$ (Figure 4 ). The observed variations in mucoadhesive performance were most likely influenced by changes in viscosity and mechanical properties of CS hydrogels during storage. Sufficiently high viscosity and preserved hydrogel structure appear to be crucial for 


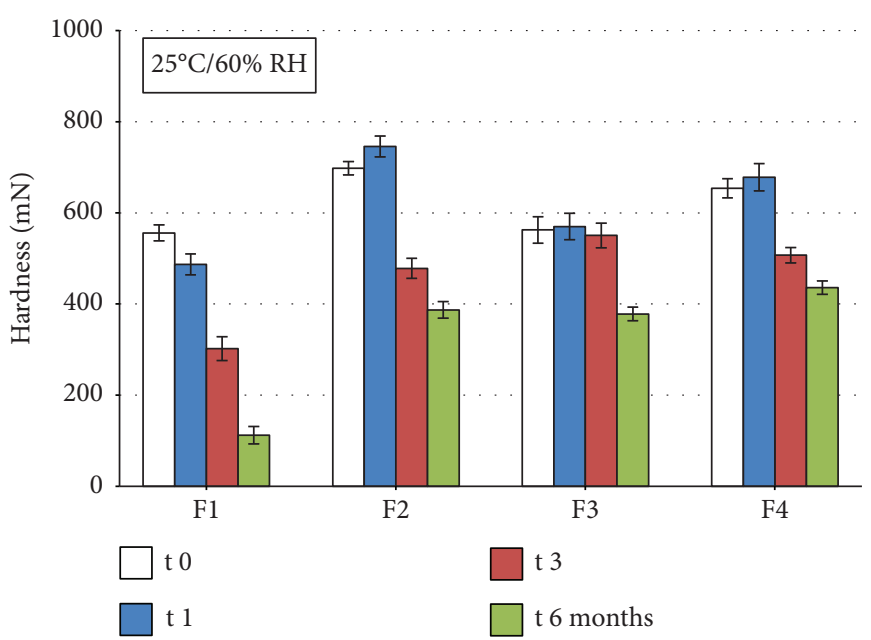

(a)

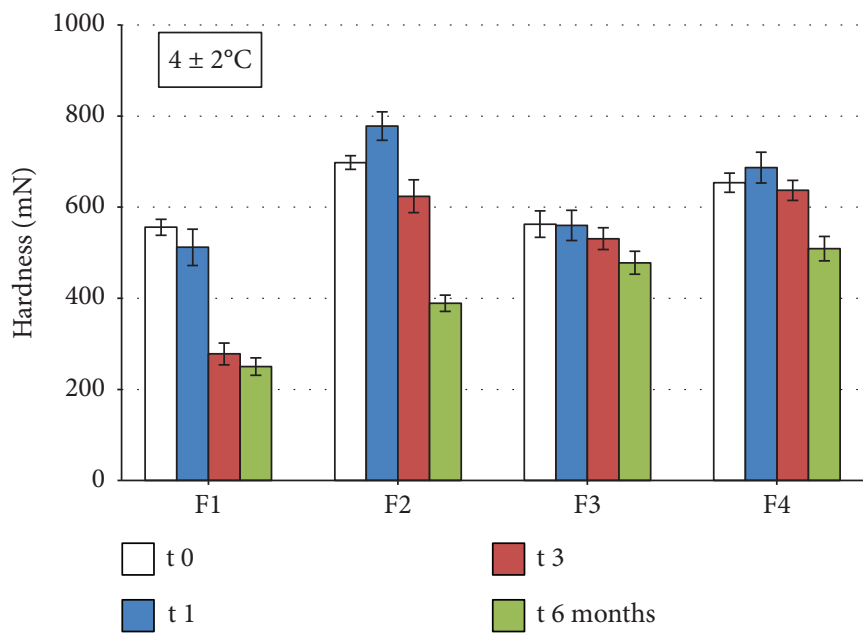

(c)

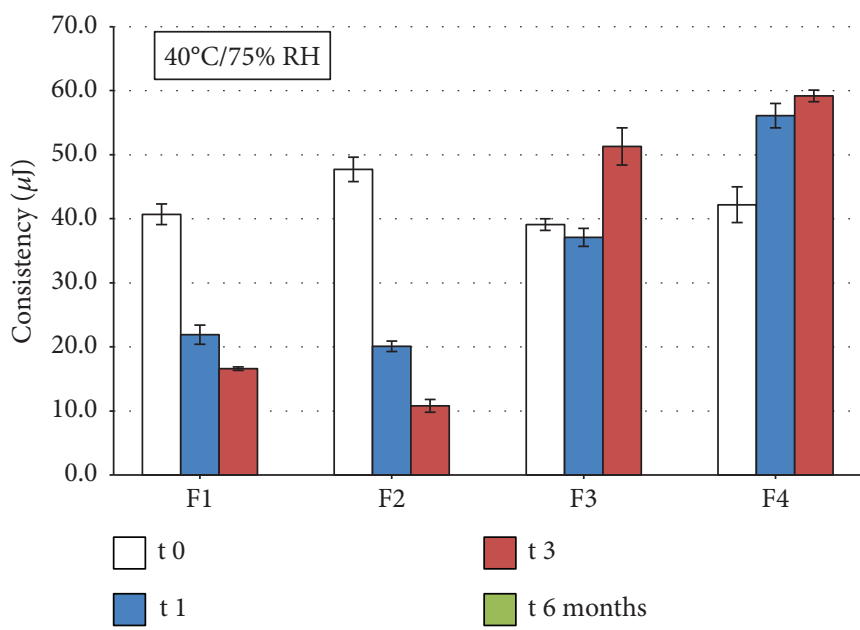

(e)

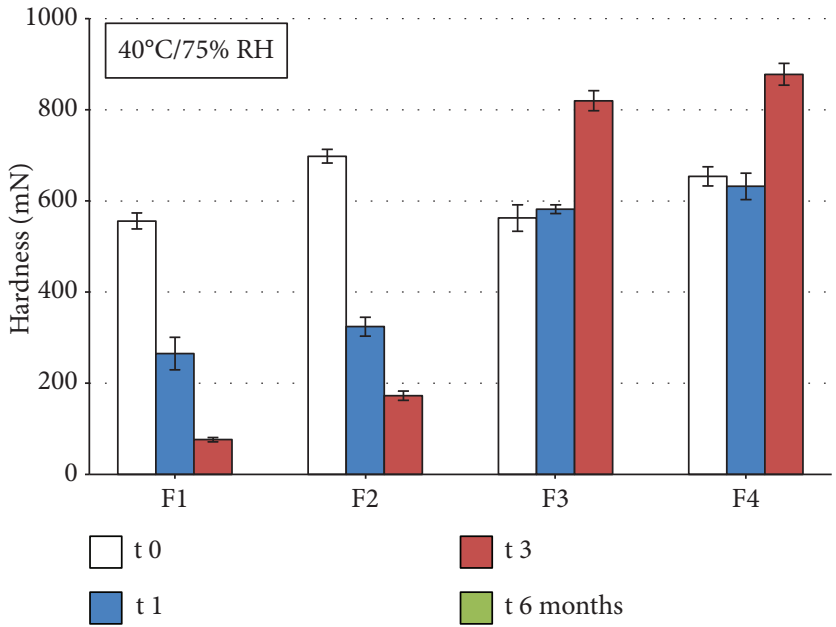

(b)

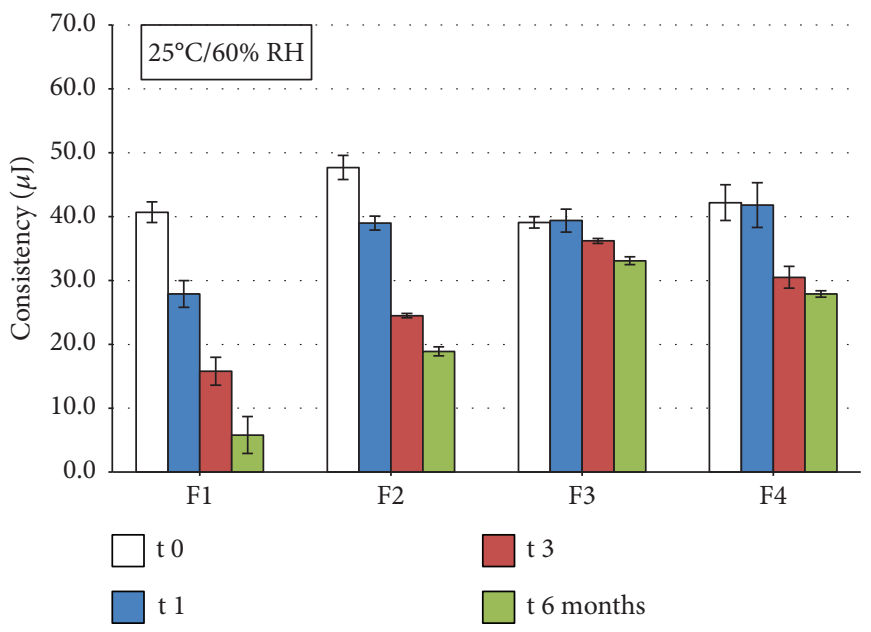

(d)

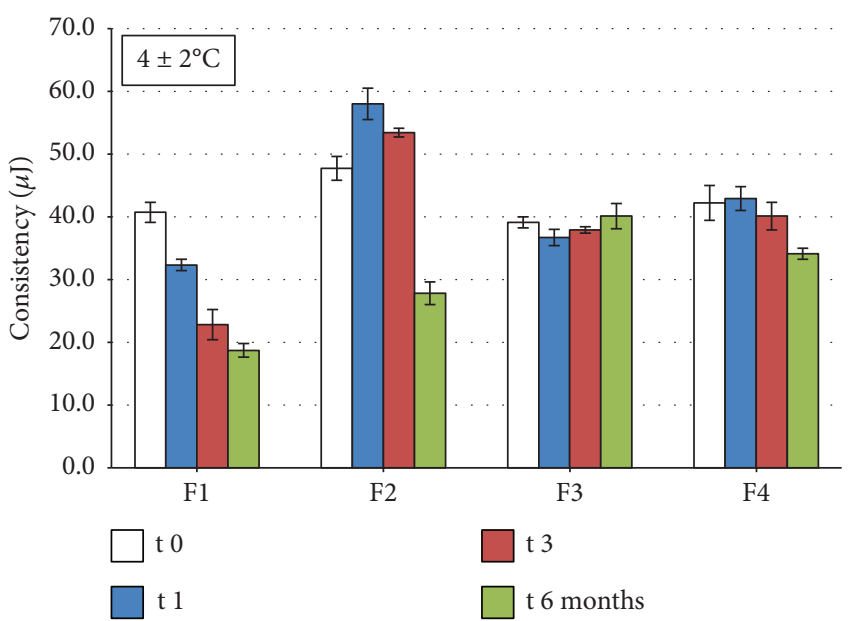

(f)

FIGURE 3: Hardness (a-c) and consistency (d-f) of hydrogels with unmodified CS (F1, F2) or with bGP-crosslinked CS (F3, F4) directly after preparation and upon 6-month storage at ambient (a,d), accelerated (b, e), and refrigerated conditions $(c, f)(n=3$, mean \pm SD).

the formation of the linkage between polymer and mucosal tissue. However, a considerable reduction in hydrogels' mucoadhesiveness could be also attributed to a decrease in their cohesive properties (defined as attractive forces existing between the hydrogel layers) resulting from loss of viscosity. 


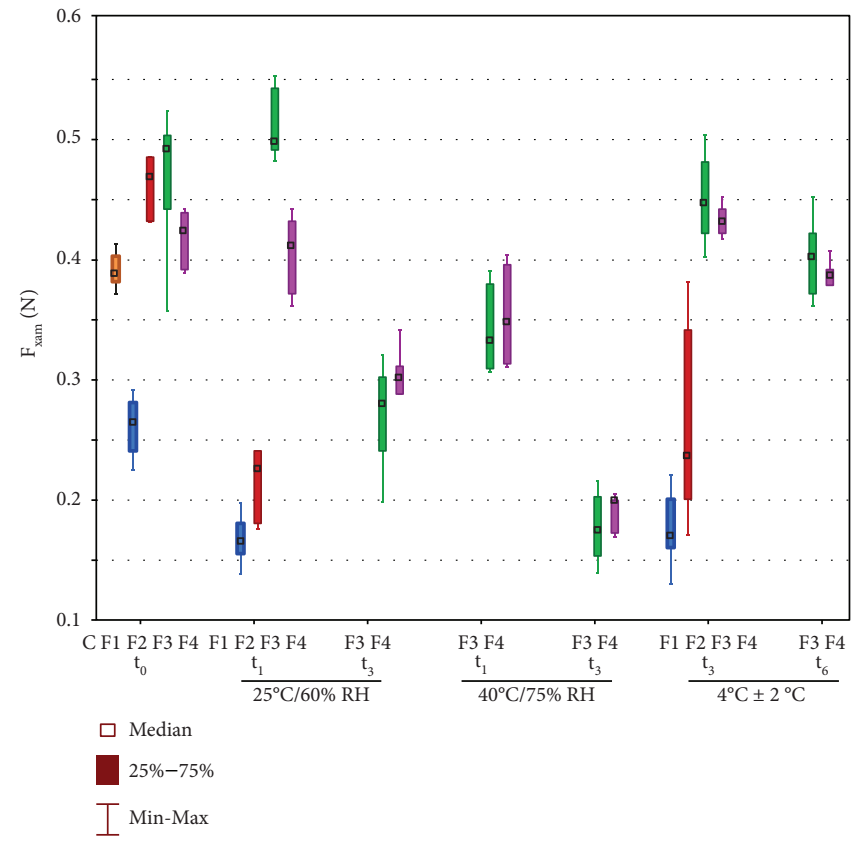

(a)

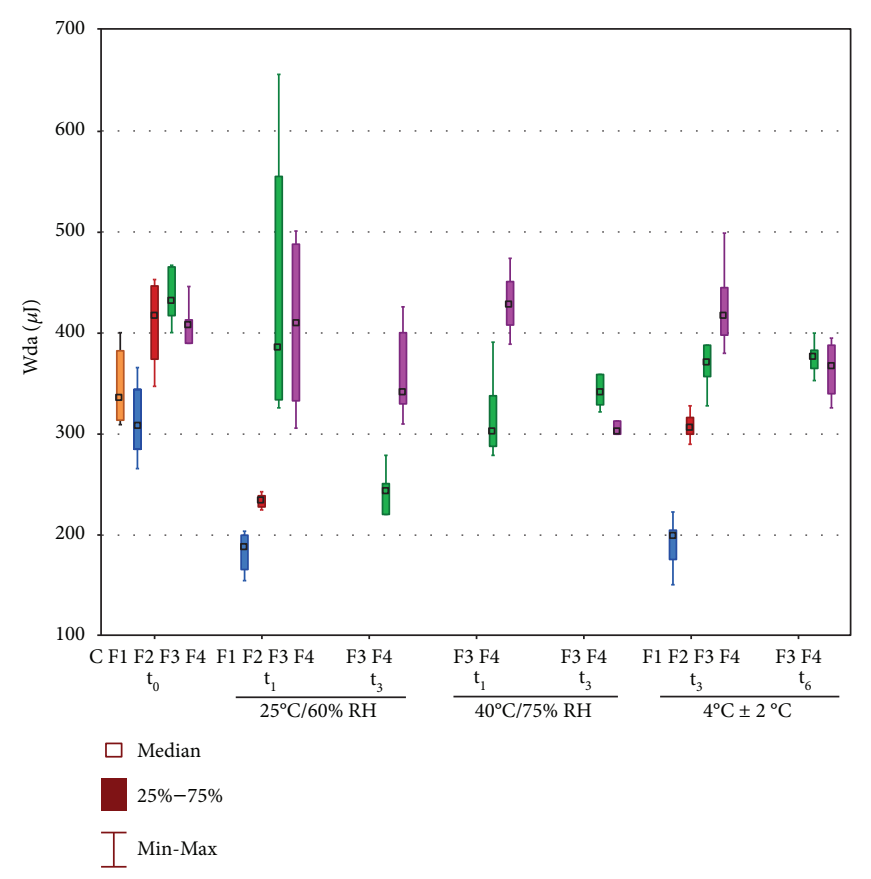

(b)

FIGURE 4: Box-plot graphs presenting mucoadhesive properties: (a) maximum force of detachment $\left(F_{\max }\right)$ and (b) work of adhesion $\left(W_{\text {ad }}\right)$ of hydrogels with unmodified CS (F1, F2) or with bGP-crosslinked CS (F3, F4) directly after preparation $\left(t_{0}\right)$ and upon 1-month $\left(t_{1}\right)$, 3-month $\left(t_{3}\right)$, or 6-month $\left(t_{6}\right)$ storage at ambient, accelerated, and refrigerated conditions $(n=6$, median); C: control, mucoadhesive vaginal gel Replens $^{\mathrm{TM}}$.

Unexpectedly, upon storage at accelerated conditions, formulations F3 and F4 were found to lose their ability to interact with mucosal tissue, regardless of simultaneous increase in their viscosity (Figure 2(b)) and mechanical properties (Figures 3(b)-3(e)). This could be attributed to the time-delayed gelation process that occurred in bGP/CS hydrogels, responsible for reduced CS chain mobility. That drop in polymer flexibility may have diminished mucoadhesive capacity of hydrogels.

3.4. In Vitro Drug Release Studies. To determine the influence of bGP crosslinking on drug release profile from CS hydrogels upon storage, dissolution tests with using the enhancer cell (equipped with Cuprophan membrane) were performed for CS formulations stored at refrigerated conditions. Because of substantial rheological changes observed for CS hydrogels upon storage at ambient and accelerated conditions, formulations F1-F4 were not analyzed in terms of drug dissolution profile. As compared to drug release rate achieved for freshly prepared formulations (Figure 5(a)), no considerable changes in drug release behavior of all hydrogels were observed after 1 month at $4 \pm 2^{\circ} \mathrm{C}$ (Figure 5(b)). However, upon 3-month storage, formulations F1 and F2 exhibited substantially faster clotrimazole release rate, most probably as a consequence of loss in hydrogels' viscosity. In contrast, hydrogels F3 and F4 stored for 3 months at $4 \pm 2{ }^{\circ} \mathrm{C}$ displayed only little alterations in drug release profile (Figure 5(c)). Substantial changes $(p<0.05)$ in drug dissolution rate from bGP/CS hydrogels occurred upon 6-month storage, and considerable differences $(p<0.05)$ in drug release curve were recorded especially with regard to formulation F3 (with lower CS concentration) (Figure 5(d)). The obtained results point on the impact of rheological characteristic of pharmaceutical semisolid dosage forms on their drug release properties.

\section{Conclusions}

The poor long-term stability of CS is a considerable drawback in the development of CS pharmaceutical preparations. In order to overcome stability challenges faced by unmodified CS formulations, physical modification (by addition of ion crosslinker) of CS hydrogels with model antifungal agent was investigated. The effect of bGP on organoleptic, physicochemical, and pharmaceutical performance of CS hydrogels was examined upon storage at ambient, accelerated, and refrigerated conditions according to ICH guideline.

A clear dependence of environmental storage conditions on CS hydrogel characteristics was displayed, and the most profound and particularly fast organoleptic and viscosity changes appeared at accelerated conditions. Notably, the introduction of bGP was shown to stabilize CS hydrogels, especially upon storage at refrigerated conditions. Formulations with bGP-crosslinked CS preserved rheological, mucoadhesive behavior, and hydrogel structure up to 3month storage at $4 \pm 2^{\circ} \mathrm{C}$. In addition, no significant alterations in physical appearance and drug content in 


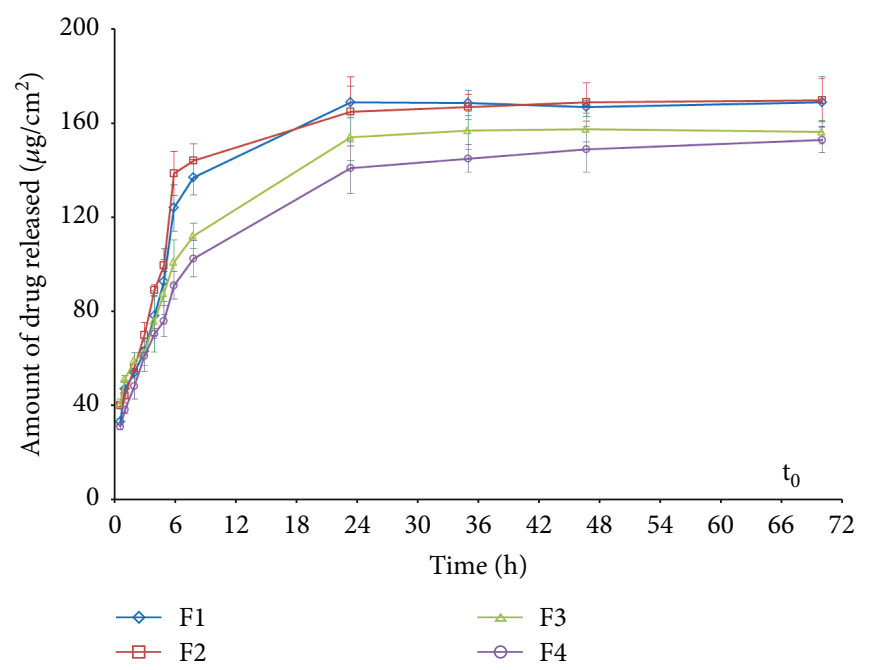

(a)

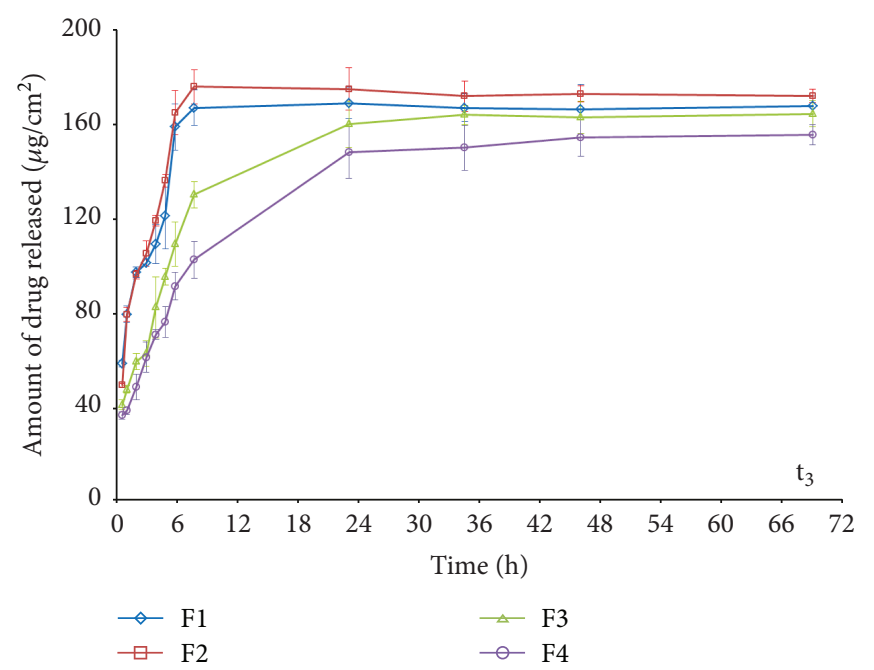

(c)

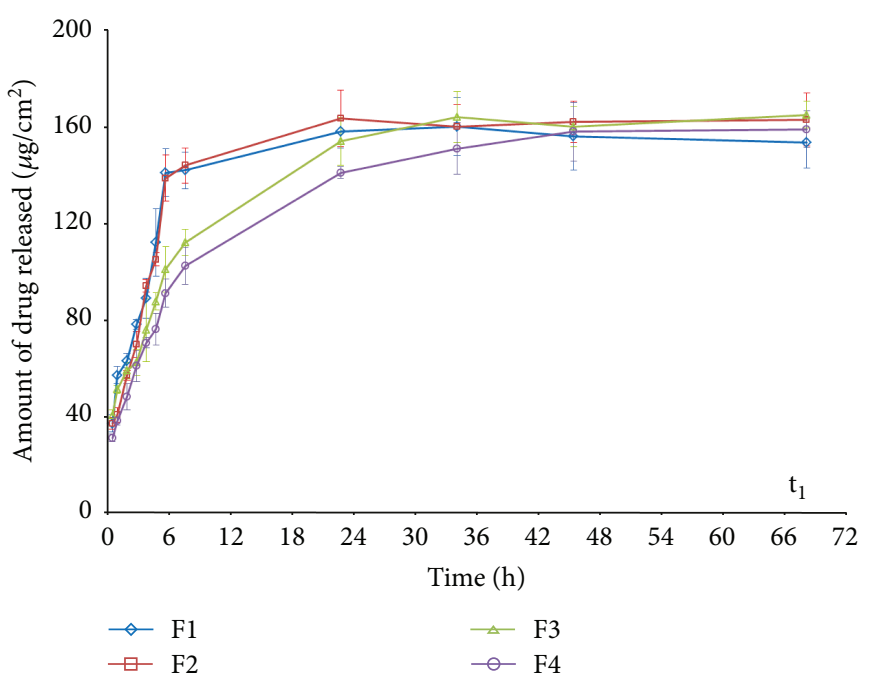

(b)

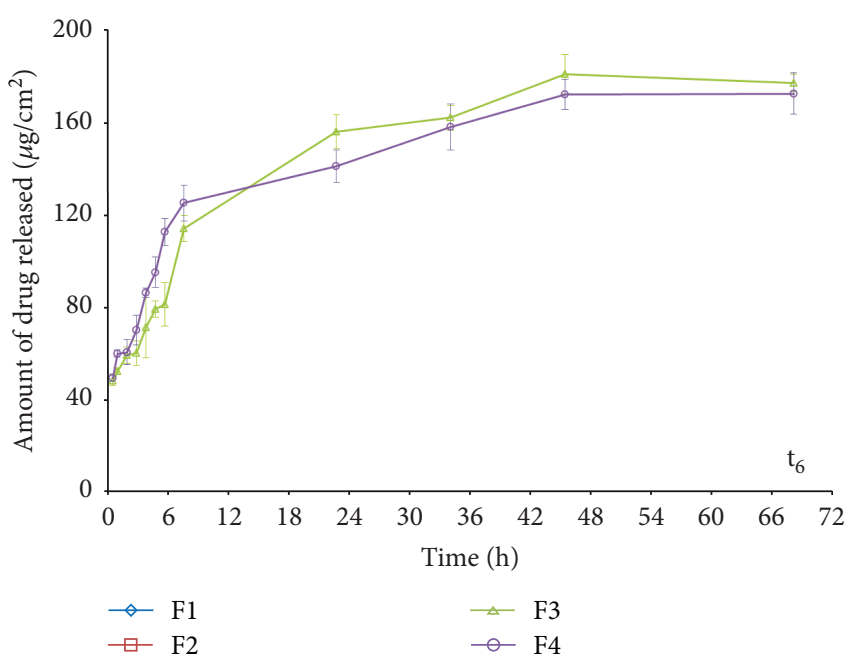

(d)

FIGURE 5: Amount of clotrimazole per unit area $\left(\mu \mathrm{g} / \mathrm{cm}^{2}\right)$ released from hydrogel with unmodified CS (F1, F2) or with bGP-crosslinked CS (F3, F4): (a) directly after preparation $\left(t_{0}\right)$ and (b) upon 1-month $\left(t_{1}\right)$, (c) 3-month $\left(t_{3}\right)$, or (d) 6-month $\left(t_{6}\right)$ storage at refrigerated conditions $(n=3$, mean $\pm \mathrm{SD})$.

formulations with bGP/CS (F3 and F4) for up to 6-month storage at $4^{\circ} \mathrm{C}$ and $25^{\circ} \mathrm{C} / 60 \% \mathrm{RH}$ were observed. In contrast, hydrogels with unmodified CS were found to have poor physicochemical stability both under ambient and refrigerated conditions, especially with regard to their viscosity and mechanical properties.

\section{Data Availability}

Access to additional data from microscopic analysis would be available upon request to the corresponding author. All other information underlying the findings of the paper is available within the manuscript.

\section{Conflicts of Interest}

The authors declare no conflict of interest.

\section{Acknowledgments}

This research work was supported by Medical University of Białystok grant (number N/ST/ZB/17/006/2215).

\section{Supplementary Materials}

The following is available as a separate file. Figure S1: optical images of hydrogels with unmodified CS (F1, F2) or with bGP-crosslinked CS (F3, F4) after preparation. Figure S2: macroscopic appearance of hydrogels with unmodified CS (F1, F2) or with bGP-crosslinked CS (F3, F4) after 6-month storage at ambient conditions. Figure S3: macroscopic appearance of hydrogels with unmodified CS (F1, F2) or with bGP-crosslinked CS (F3, F4) after 3-month storage at accelerated conditions. Figure S4: macroscopic appearance of hydrogels with unmodified CS (F1, F2) or with bGP- 
crosslinked CS (F3, F4) after 6-month storage at refrigerated conditions. (Supplementary Materials)

\section{References}

[1] A. Srivastava, T. Yadav, S. Sharma, A. Nayak, A. Akanksha Kumari, and N. Mishra, "Polymers in drug delivery," Journal of Biosciences and Medicines, vol. 4, no. 1, pp. 69-84, 2016.

[2] J. R. Franca, G. Foureaux, L. L. Fuscaldi et al., "Bimatoprostloaded ocular inserts as sustained release drug delivery systems for glaucoma treatment: in vitro and in vivo evaluation," PLoS One, vol. 9, no. 4, pp. e95461-e95472, 2014.

[3] F. Saporito, G. Sandri, S. Rossi et al., "Freeze dried chitosan acetate dressings with glycosaminoglycans and traxenamic acid," Carbohydrate Polymers, vol. 184, pp. 408-417, 2018.

[4] J. Venkatesan, I. Bhatnagar, and S. K. Kim, "Chitosan-alginate biocomposite containing fucoidan for bone tissue engineering," Marine Drugs, vol. 12, no. 1, pp. 300-316, 2014.

[5] B. Choi, S. Kim, B. Lin, B. M. Wu, and M. Lee, "Cartilaginous extracellular matrix-modified chitosan hydrogels for cartilage tissue engineering," ACS Applied Materials \& Interfaces, vol. 6, no. 22, pp. 20110-20121, 2014.

[6] Y. H. Lee, H. I. Park, and J. S. Choi, "Novel glycol chitosanbased polymeric gene carrier synthesized by a Michael addition reaction with low molecular weight polyethylenimine," Carbohydrate Polymers, vol. 137, pp. 669-677, 2016.

[7] A. Smith, M. Perelman, and M. Hinchcliffe, "Chitosan: a promising safe and immune-enhancing adjuvant for intranasal vaccines," Human Vaccines \& Immunotherapeutics, vol. 10, no. 3, pp. 797-807, 2014.

[8] P. N. Vaingankar and A. R. Juvekar, "Fermentative production of mycelial chitosan from Zygomycetes: media optimization and physico-chemical characterization," Advances in Bioscience and Biotechnology, vol. 05, no. 12, pp. 940-956, 2014.

[9] T. A. Ahmed and B. M. Aljaeid, "Preparation, characterization, and potential application of chitosan, chitosan derivatives, and chitosan metal nanoparticles in pharmaceutical drug delivery," Drug Design, Development and Therapy, vol. 10, pp. 483-507, 2016.

[10] I. Aranaz, N. Acosta, C. Civera et al., "Cosmetics and cosmeceutical applications of chitin, chitosan and their derivatives," Polymer, vol. 10, no. 2, p. 213, 2018.

[11] T. T. Nguyen, A. R. Barber, K. Corbin, and W. Zhang, "Lobster processing by-products as valuable bioresource of marine functional ingredients, nutraceuticals, and pharmaceuticals," Bioresources and Bioprocessing, vol. 4, no. 1, pp. 27-46, 2017.

[12] S. Baltzley, A. Mohammad, A. H. Malkawi, and A. M. AlGhananeem, "Intranasal drug delivery of olanzapine-loaded chitosan nanoparticles," AAPS PharmSciTech, vol. 15, no. 6, pp. 1598-1602, 2014.

[13] G. Tejada, M. G. Barrera, G. N. Piccirilli et al., "Development and evaluation of buccal films based on chitosan for the potential treatment of oral candidiasis," AAPS PharmSciTech, vol. 18, no. 4, pp. 936-946, 2017.

[14] A. Almomen, S. Cho, C. H. Yang et al., "Thermosensitive progesterone hydrogel: a safe and effective new formulation for vaginal application," Pharmaceutical Research, vol. 32, no. 7, pp. 2266-2279, 2015.

[15] E. Szymańska, K. Winnicka, A. Amelian, and U. Cwalina, "Vaginal chitosan tablets with clotrimazole-design and evaluation of mucoadhesive properties using porcine vaginal mucosa, mucin and gelatine," Chemical and Pharmaceutical Bulletin, vol. 62, no. 2, pp. 160-167, 2014.

[16] F. Notario-Pérez, A. Martín-Illana, R. Cazorla-Luna et al., "Influence of chitosan swelling behaviour on controlled release of tenofovir from mucoadhesive vaginal systems for prevention of sexual transmission of HIV," Marine Drugs, vol. 15, no. 2, pp. 50-66, 2017.

[17] I. Younes, S. Sellimi, M. Rinaudo, K. Jellouli, and M. Nasri, "Influence of acetylation degree and molecular weight of homogeneous chitosans on antibacterial and antifungal activities," International Journal of Food Microbiology, vol. 185, pp. 57-63, 2014.

[18] M. Egusa, R. Iwamoto, H. Izawa et al., "Characterization of chitosan nanofiber sheets for antifungal application," International Journal of Molecular Sciences, vol. 16, no. 11, pp. 2620226210, 2015.

[19] E. Szymańska, K. Winnicka, P. Wieczorek, P. Sacha, and E. Tryniszewska, "Influence of unmodified and $\beta$ glycerophosphate cross-linked chitosan on anti-Candida activity of clotrimazole in semi-solid delivery systems," International Journal of Molecular Sciences, vol. 15, no. 10, pp. 17765-17777, 2014.

[20] E. Szymańska, K. Sosnowska, W. Miltyk, M. Rusak, A. Basa, and K. Winnicka, "The effect of $\beta$-glycerophosphate crosslinking on chitosan cytotoxicity and properties of hydrogels for vaginal application," Polymer, vol. 7, no. 11, pp. 2223-2244, 2015.

[21] E. Szymańska and K. Winnicka, "Stability of chitosan-a challenge for pharmaceutical and biomedical applications," Marine Drugs, vol. 13, no. 4, pp. 1819-1846, 2015.

[22] M. Fernandes, I. C. Gonçalves, S. Nardecchia, I. F. Amaral, M. A. Barbosa, and M. C. L. Martins, "Modulation of stability and mucoadhesive properties of chitosan microspheres for therapeutic gastric application," International Journal of Pharmaceutics, vol. 454, no. 1, pp. 116-124, 2013.

[23] C. Liu, E. Thormann, P. M. Claesson, and E. Tyrode, "Surface grafted chitosan gels. Part II. Gel formation and characterization," Langmuir, vol. 30, no. 29, pp. 8878-8888, 2014.

[24] A. N. Allam, V. F. Naggar, and S. S. El Gamal, "Formulation and physicochemical characterization of chitosan/acyclovir co-crystals," Pharmaceutical Development and Technology, vol. 18, no. 4, pp. 856-865, 2013.

[25] M. Rinaudo, G. Pavlov, and J. Desbrières, "Influence of acetic acid concentration on the solubilization of chitosan," Polymer, vol. 40, no. 25, pp. 7029-7032, 1999.

[26] "International conference on harmonisation of technical requirements for registration of pharmaceuticals for human use, ICH harmonised tripartite guideline, evaluation for stability data Q1E,” April 2018, http://www.ich.org/fileadmin/ Public.../Guidelines/.../Q1E_Guideline.pdf.

[27] The European Pharmacopoeia 7.0, Council of Europe, Strasbourg, France, 2011.

[28] EMA, ICH Topic Q4B Annex 12 Analytical Sieving General ChapterMarch 2018, http://www.ema.europa.eu/docs/en_GB/ document_library/Scientific_guideline/2010/01/WC500044305. pdf.

[29] "In vitro release testing and in vivo bioequivalence documentation," in Guidance for Industry: SUPAC-SS Non-Sterile Semisolid Dosage Forms. Scale-up and Postapproval Changes: 
Chemistry, Manufacturing and Controls, pp. 19-24, FDASUPAC-SS, Rockville, MD, USA, 1999.

[30] A. Chenite, M. Buschmann, D. Wang, C. Chaput, and N. Kandani, "Rheological characterisation of thermogelling chitosan/glycerol-phosphate solutions," Carbohydrate Polymers, vol. 46, no. 1, pp. 39-47, 2001.

[31] The United States Pharmacopeia USP 34-NF 29, The United States Pharmacopeial Convention, Rockville, MD, USA, 2011.

[32] W. Y. Chien, "Drug delivery: vaginal route," in Encyclopedia of Pharmaceutical Technology, vol. 2, J. Swarbrick, Ed., pp. 13391361, Informa Healthcare, New York, NY, USA, 3rd edition, 2007.

[33] S. E. Harding, "Some observations on the effects of bioprocessing on biopolymer stability," Journal of Drug Targeting, vol. 18, no. 10, pp. 732-740, 2010.

[34] D. P. Chattopadhyay and M. S. Inamdar, "Aqueous behaviour of chitosan," International Journal of Polymer Science, vol. 2010, Article ID 939536, 7 pages, 2010.

[35] T. T. B. Nguyen, S. Hein, C. H. Ng, and W. F. Stevens, "Molecular stability of chitosan in acid solutions stored at various conditions," Journal of Applied Polymer Science, vol. 107, no. 4, pp. 2588-2593, 2008.

[36] B. Martini, S. Dimida, E. de Benedetto, M. Madaghiele, and C. Demitri, "Study on the degradation of chitosan slurries," Results in Physics, vol. 6, pp. 728-729, 2016.

[37] E. Ruel-Gariépy, A. Chenite, C. Chaput, S. Guirguis, and J.-C. Leroux, "Characterization of thermosensitive chitosan gels for the sustained delivery of drugs," International Journal of Pharmaceutics, vol. 203, no. 1-2, pp. 89-98, 2000.

[38] S. Supper, N. Anton, N. Seidel, M. Riemenschnitter, C. Schoch, and T. Vandamme, "Rheological study of chitosan/polyol-phosphate systems: influence of the polyol part on the thermo-induced gelation mechanism," Langmuir, vol. 29, no. 32, pp. 10229-10237, 2013. 


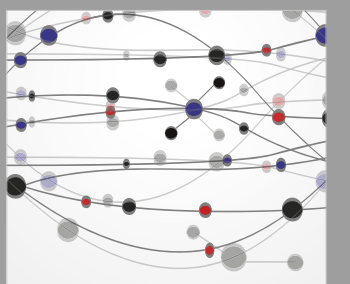

The Scientific World Journal
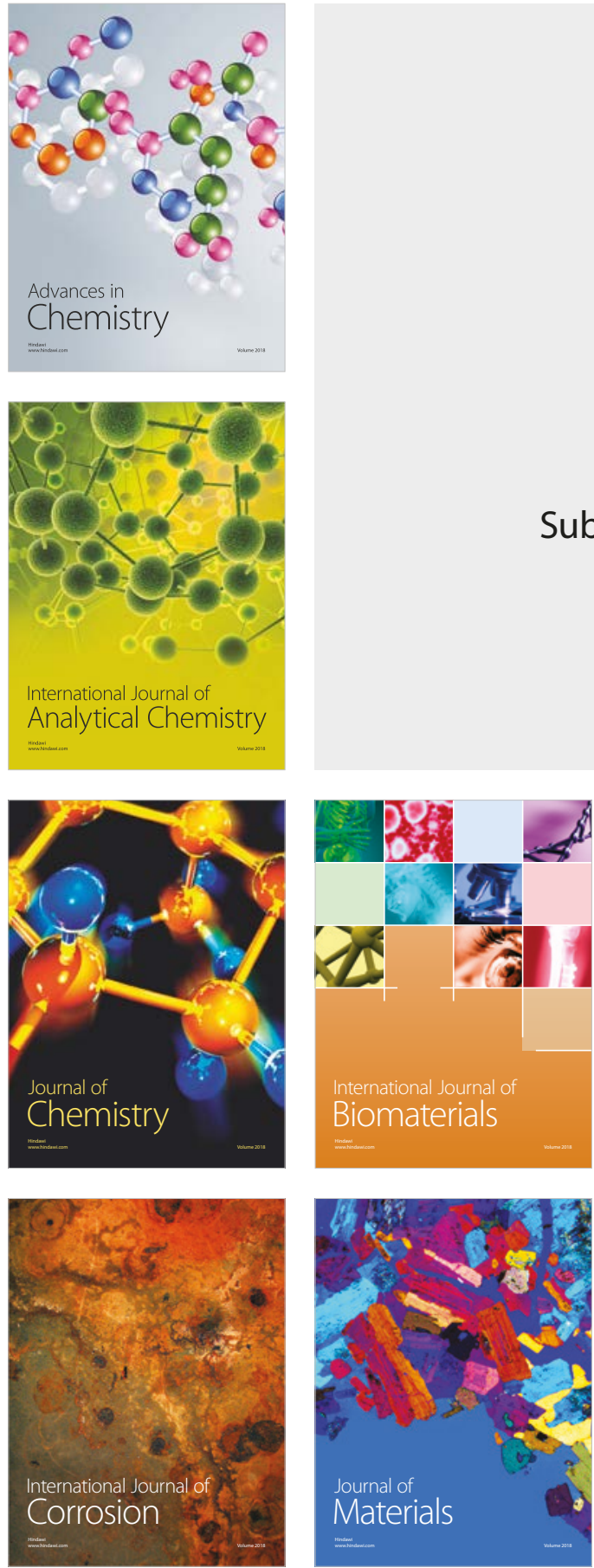

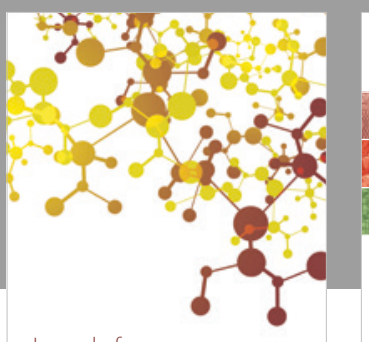

Journal of

Applied Chemistry
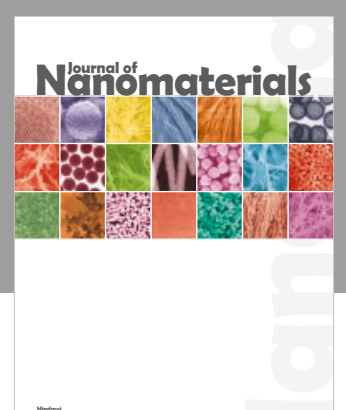

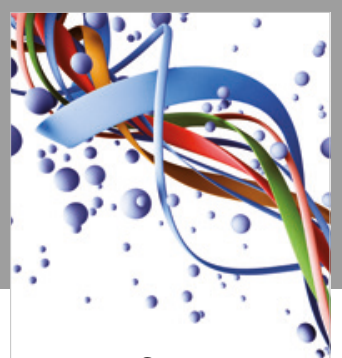

Scientifica

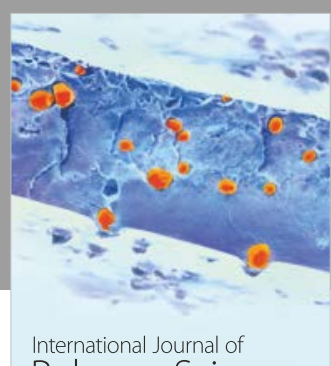

Polymer Science

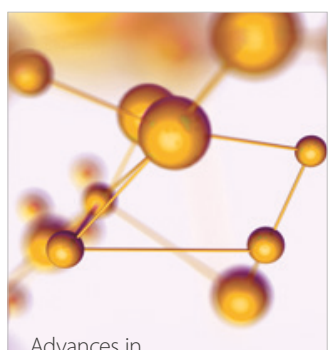

Physical Chemistry
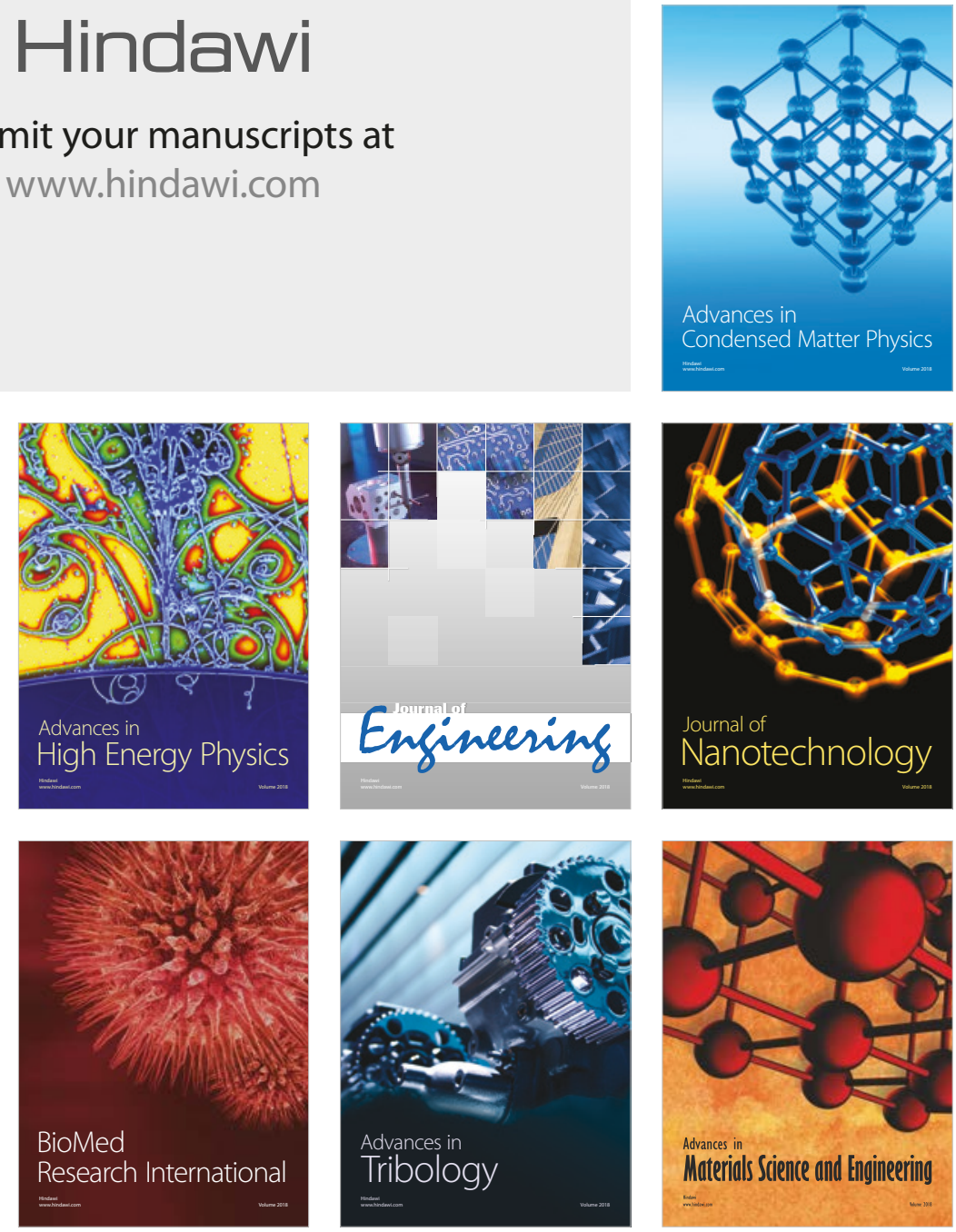